九州大学学術情報リポジトリ

Kyushu University Institutional Repository

Chlamydia pneumoniae exploits adipocyte lipid chaperone FABP4 to facilitate fat mobilization and intracellular growth in murine adipocytes

ニラワナ, フィテリアニ, ワレナ

https://doi. org/10.15017/1931804

出版情報：九州大学，2017，博士（医学），課程博士

バージョン：

権利関係：๑2017 Elsevier Inc. All rights reserved 


\title{
Chlamydia pneumoniae exploits adipocyte lipid chaperone FABP4 to facilitate fat mobilization and intracellular growth in murine adipocytes
}

\author{
Nirwana Fitriani Walenna ${ }^{\mathrm{a}, \mathrm{b}}$, Yusuke Kurihara ${ }^{\mathrm{a}}$, Bin Chou ${ }^{\mathrm{a}}$, Kazunari Ishii ${ }^{\mathrm{a}}$, \\ Toshinori Soejima ${ }^{\mathrm{a}}$, Ryota Itoh ${ }^{\mathrm{a}}$, Akinori Shimizu ${ }^{\mathrm{a}}$, Takeshi Ichinohe ${ }^{\mathrm{c}}$, \\ Kenji Hiromatsu ${ }^{\text {a,* }}$ \\ a Department of Microbiology \& Immunology, Faculty of Medicine, Fukuoka University, Fukuoka 814-0180, Japan \\ ${ }^{\mathrm{b}}$ Department of Bacteriology, Graduate School of Medical Sciences, Kyushu University, Fukuoka 812-8582, Japan \\ c Division of Viral Infection, International Research Center for Infectious Diseases, Institute of Medical Science, University of Tokyo, Tokyo 108-8639, Japan
}

\section{A R T I C L E I N F O}

\section{Article history:}

Received 23 October 2017

Accepted 2 November 2017

Available online 3 November 2017

\section{Keywords:}

Chlamydia pneumoniae

Adipocytes

Infection

Lipolysis

Fatty acid-binding protein 4

\begin{abstract}
A B S T R A C T
Fatty acid-binding protein 4 (FABP4), a cytosolic lipid chaperone predominantly expressed in adipocytes and macrophages, modulates lipid fluxes, trafficking, signaling, and metabolism. Recent studies have demonstrated that FABP4 regulates metabolic and inflammatory pathways, and in mouse models its inhibition can improve type 2 diabetes mellitus and atherosclerosis. However, the role of FABP4 in bacterial infection, metabolic crosstalk between host and pathogen, and bacterial pathogenesis have not been studied. As an obligate intracellular pathogen, Chlamydia pneumoniae needs to obtain nutrients such as ATP and lipids from host cells. Here, we show that $C$. pneumoniae successfully infects and proliferates in murine adipocytes by inducing hormone sensitive lipase (HSL)-mediated lipolysis. Chemical inhibition or genetic manipulation of HSL significantly abrogated the intracellular growth of C. pneumoniae in adipocytes. Liberated free fatty acids were utilized to generate ATP via $\beta$-oxidation, which C. pneumoniae usurped for its replication. Strikingly, chemical inhibition or genetic silencing of FABP4 significantly abrogated $C$. pneumoniae infection-induced lipolysis and mobilization of liberated FFAs, resulting in reduced bacterial growth in adipocytes. Collectively, these results demonstrate that C. pneumoniae exploits host FABP4 to facilitate fat mobilization and intracellular replication in adipocytes. This work uncovers a novel strategy used by intracellular pathogens for acquiring energy via hijacking of the host lipid metabolism pathway.
\end{abstract}

๑) 2017 Elsevier Inc. All rights reserved.

\section{Introduction}

Adipocyte fatty acid-binding protein (FABP4, also known as aP2/ A-FABP) is an intracellular lipid chaperone abundantly expressed in adipocytes and macrophages [1]. By binding with long-chain fatty acids, FABP4 can affect the uptake, transportation, esterification, and $\beta$-oxidation of fatty acids and regulate the energy balance and lipid signal transduction within cells [1,2]. Evidence suggests that

Abbreviations: ATGL, adipose triglyceride lipase; HSL, hormone sensitive lipase; MGL, monoglyceride lipase; FABP4, fatty acid binding protein 4; FFAs, free fatty acids; LD, lipid droplet; CPT-1, carnitine palmitoyltransferase 1; FAO, fatty acid oxidation; OCR, oxygen consumption rate.

* Corresponding author.

E-mail address: khiromatsu@fukuoka-u.ac.jp (K. Hiromatsu).
FABP4 is central to the generation of meta-inflammation, especially upon exposure to lipids, and couples lipotoxicity to organelle function and endoplasmic reticulum (ER) stress; furthermore, FABP4 inhibition improved type 2 diabetes mellitus and atherosclerosis in mouse models [3-7]. In humans, elevated circulating FABP4 levels are associated with obesity and metabolic diseases $[8,9]$. Thus, targeting FABP4 offers a novel therapeutic approach for the treatment of many metabolic diseases [10]. However, the roles of FABP4 in bacterial infection, metabolic crosstalk between host and pathogen have not been studied.

Chlamydia pneumoniae is an obligate intracellular pathogen that needs to obtain nutrients, such as nucleotides (ATP), amino acids, and lipids, from host cells for its replication [11,12]. To meet their bioenergetic and biosynthetic demands for replication, intracellular pathogens modulate host metabolism. However, how the pathogen 
A 3T3-L1 Preadipocyte Mock

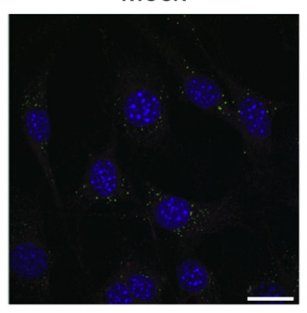

D

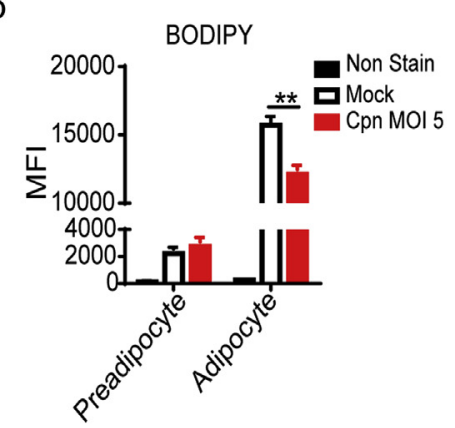

B 3T3-L1 purified re-plated adipocyte

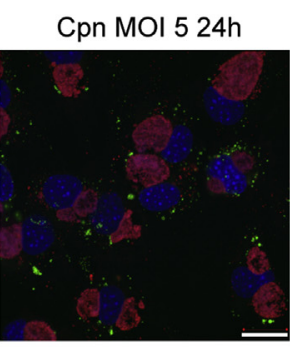

E

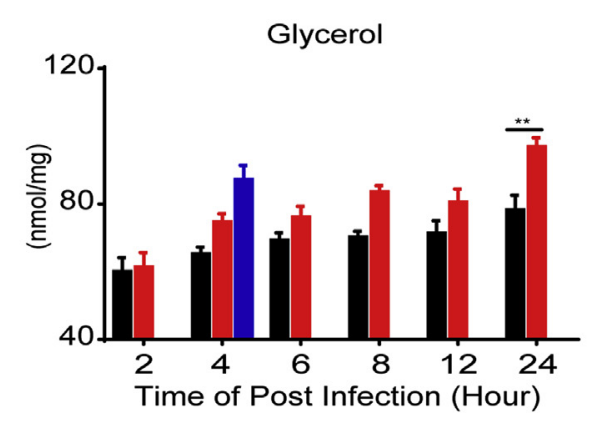

Cpn MOI $524 \mathrm{~h}$

Cpn LPS BODIPY Hoechst

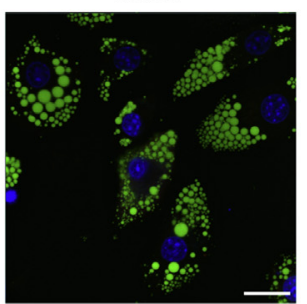

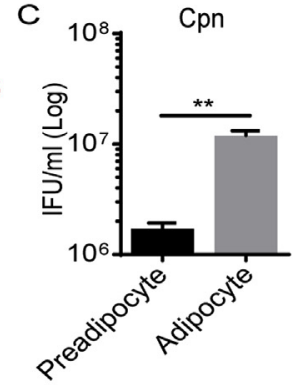
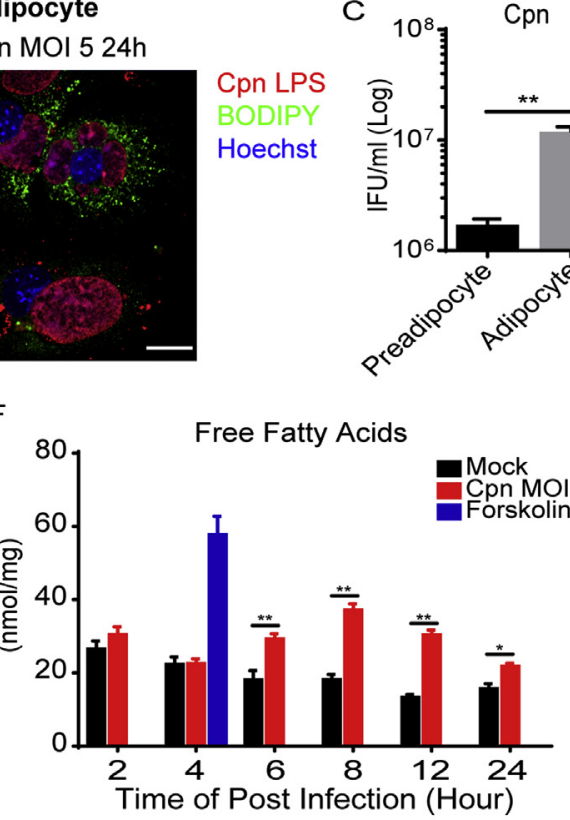

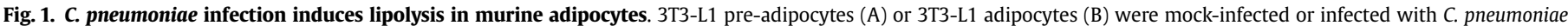

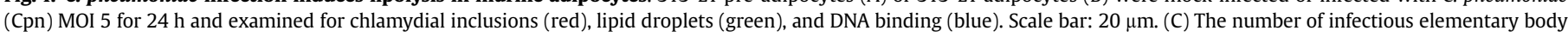

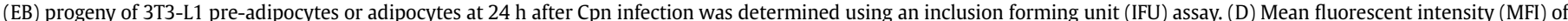

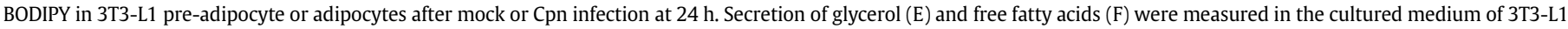

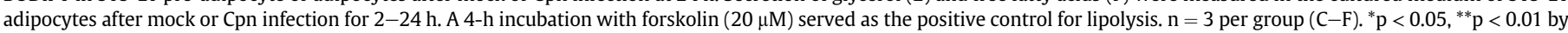
Student $t$-test (C) or two-way ANOVA (D-F).

modulates host metabolism to meet its bioenergetic demands for growth is largely unknown. Adipose tissues may provide a niche or reservoir for intracellular pathogens like Mycobacterium tuberculosis [13], Rickettsia prowazekii [14], and Trypanosoma cruzi [15]. Adipocytes may provide a suitable host for obligate intracellular pathogens, which require various nutrients or energy source from host cells [16]. It has been demonstrated that $C$. pneumoniae can infect murine pre- and post-differentiated adipocytes and, through a TNF- $\alpha$ mediated inflammatory mechanism, can impair differentiation and insulin signaling [17]. However, how C. pneumoniae proliferates in adipocytes and how the abundance of lipid stores in adipocytes may favor its survival, growth, and persistence remains unexplored topics.

Herein, we demonstrate that $C$. pneumoniae successfully infects mature adipocytes and induces hormone-sensitive lipase (HSL)mediated lipolysis. In addition, we demonstrate that liberated free fatty acids (FFAs) undergo $\beta$-oxidation and generate ATP in host adipocytes, which $C$. pneumoniae usurps for its intracellular propagation. Collectively, our data demonstrate that $C$. pneumoniae reprograms lipid metabolism by hijacking FABP4 for its own benefit and sheds new light on the etiological link between $C$. pneumoniae infection and metabolic syndrome.

\section{Materials and methods}

\subsection{Microbes}

C. pneumoniae (strain AR39, ATCC53592) and C. muridarum (mouse pneumonitis strain Nigg II, ATCC VR-123) were obtained from the ATCC and propagated as previously described [18].

\subsection{T3-L1 adipocyte differentiation}

3T3-L1 preadipocytes (ATCC CL-173) or stable shRNA- knockdown (control EGFP, HSL) preadipocytes were seeded in 24well plates and allowed to reach confluence for 2 days. On day 0 , adipocyte differentiation was induced by adding $2.5 \mu \mathrm{M}$ dexamethasone, $0.5 \mathrm{mM}$ 3-isobutyl-1-methylexanthine (IBMX), and $10 \mu \mathrm{g} / \mathrm{mL}$ insulin. On day 2 and thereafter, the medium was replaced with DMEM-10\% FCS containing only $10 \mu \mathrm{g} / \mathrm{mL}$ insulin.

\subsection{In vitro infection of adipocytes}

Cultured adipocytes were infected at days 4-6 of differentiation. To initiate infection, $C$. pneumoniae or $C$. muridarum was added to plates at $\mathrm{MOI}=5$, and plates were centrifuged at $900 \times \mathrm{g}$ and $25{ }^{\circ} \mathrm{C}$ for $1 \mathrm{~h}$. Next, the inoculum was removed, and cells were cultured in DMEM containing 10\% FCS and streptomycin $(100 \mu \mathrm{g} /$ $\mathrm{mL}$ ). Inhibitors were added $60 \mathrm{~min}$ after inoculation with C. pneumoniae or C. muridarum.

\subsection{IFU assay}

Infected cells were collected, frozen and thawed, serially diluted 10-fold in SPG medium, and reseeded into 24-well plates containing a HeLa-cell monolayer. After centrifugation at $900 \times g$ and $25^{\circ} \mathrm{C}$ for $1 \mathrm{~h}$, and cells were cultured in DMEM containing 10\% FCS and $1 \mu \mathrm{g} / \mathrm{mL}$ cycloheximide. After 24-48 h, cells were fixed with ice-cold methanol for $10 \mathrm{~min}$ and stained with a FITC-conjugated anti-Chlamydia LPS-specific monoclonal antibody (PROGEN).

\subsection{Immunofluorescence}

Chlamydia-infected preadipocytes or adipocytes were fixed with $4 \%$ paraformaldehyde, blocked and permeabilized with $1 \%$ BSA and 1\% Triton X-100 in PBS for 10 min at RT and incubated with avidin/ biotin blocking buffer (abcam). Next, the cells were incubated with primary antibodies against biotin conjugated anti-chlamydial LPS 
A

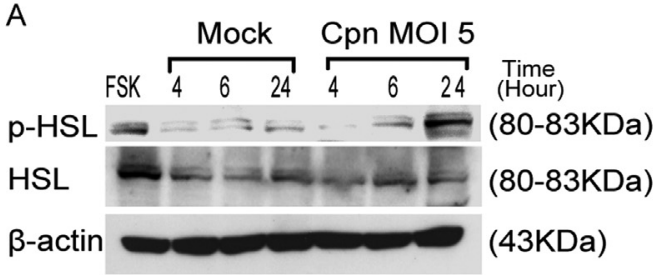

\section{B}

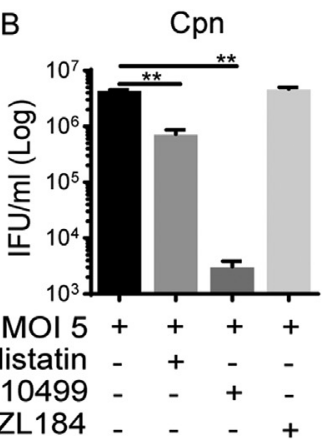

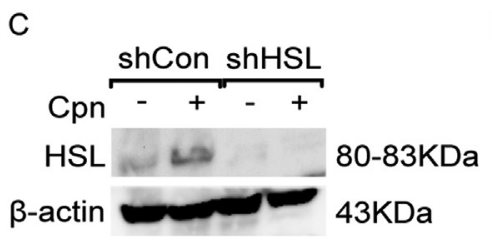

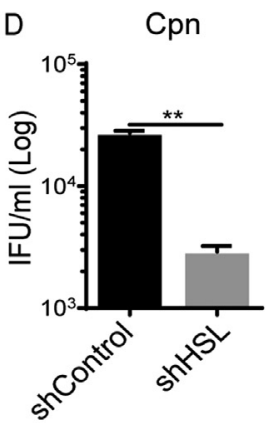

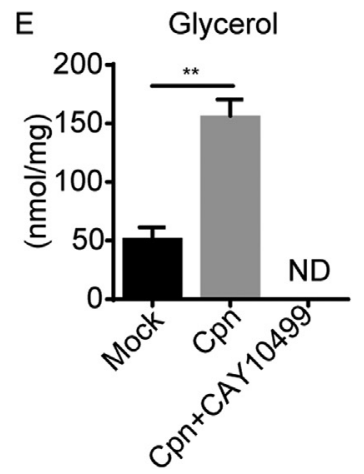

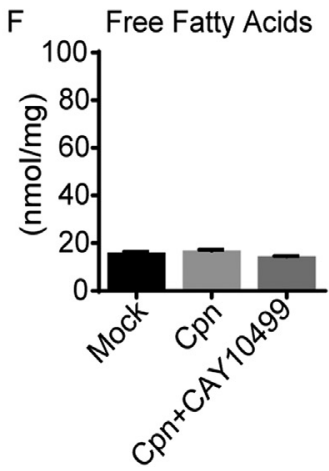

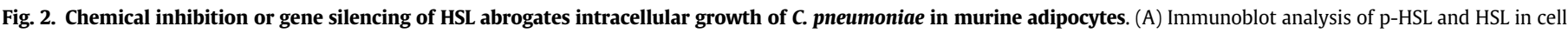

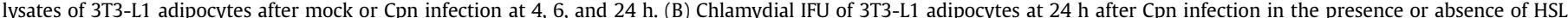

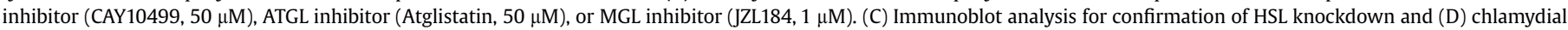

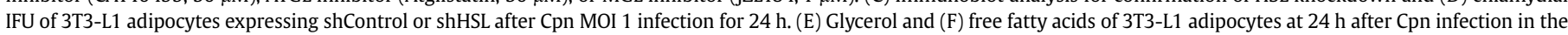
presence or absence of HSL inhibitors, ND = not detected. $n=3$ per group (B, D-F) ${ }^{* *} \mathrm{p}<0.01$, one-way ANOVA (B, E); Student $t$-test (D).

(GeneTex) for $1 \mathrm{~h}$ and Alexa Fluor 555-labeled secondary antibodies (Invitrogen), BODIPY493/503 (Invitrogen), and Hoechst 33342 (Thermo Fisher) for $30 \mathrm{~min}$. Stained coverslips were mounted with Prolong Gold Antifade Mountant (Life Technologies). Images were acquired using a LSM 710 (Zeiss).

\subsection{Immunoblot}

Cells were washed once in ice-cold PBS, lysed in RIPA buffer with a protease inhibitor cocktail (Nacalai Tesque) and PhosSTOP phosphatase inhibitor cocktail (Roche), and then briefly sonicated. Membranes were blocked in Blocking One (Nacalai Tesque) for $30 \mathrm{~min}$ and incubated with primary antibody diluted in Can Get Signal solution 1 (TOYOBO) overnight at $4{ }^{\circ} \mathrm{C}$ and then with horseradish peroxidase (HRP)-conjugated secondary antibodies to rabbit/mouse IgGs (GE Healthcare) diluted in Can Get Signal solution 2 (TOYOBO) for $1 \mathrm{~h}$ at RT. Immunoreactive bands were detected by ECL Blotting Reagents (GE Healthcare).

\subsection{Oxygen Consumption Rate (OCR) assay}

The OCR was measured using the MitoXpress Xtra Oxygen Consumption Assay (Luxcel Biosciences). MitoXpress fluorescence analysis was performed using a Tecan Infinite 200 Pro microplate reader.

\subsection{Statistics}

Results are expressed as the mean \pm SEM and are representative of at least three experiments. Data analyses were performed using the GraphPad Prism software version 6.0. All $\mathrm{P}<0.05$ were considered statistically significant.
The detail information on material and methods were provided in Supplementary methods.

\section{Results}

\subsection{C. pneumoniae infection induces lipolysis in murine adipocytes}

We observed that $C$. pneumoniae successfully infects and proliferates in differentiated 3T3-L1 mouse adipocytes. Sizes of chlamydial inclusion bodies in differentiated adipocytes were larger than those of pre-adipocytes (Fig. 1A and Fig. 1B). The number of infectious progeny in adipocytes, judged by an inclusion forming unit (IFU) assay, was higher than that of pre-adipocytes (Fig. 1C). Strikingly, after $C$. pneumoniae infection, the chlamydial inclusion body developed with a simultaneous reduction in the number of lipid droplets in adipocytes (Fig. 1B). Flow cytometry using the fluorescent probe BODIPY to detect neutral lipids confirmed that C. pneumoniae infection in adipocytes induced a significant reduction in the BODIPY signal (Fig. 1D). Notably, we observed that C. pneumoniae infection induced a significant increase in glycerol and fatty acid release from adipocytes, indicating that C. pneumoniae infection induces lipolysis in murine adipocytes (Fig. 1E and F).

\subsection{Chemical inhibition or genetic manipulation of HSL abrogates C. pneumoniae infection-induced lipolysis and intracellular growth of bacteria in adipocytes}

Next, we examined the mechanism underlying $C$. pneumoniae infection-induced lipolysis in adipocytes. Hormone sensitive lipase (HSL) and adipose triglyceride lipase (ATGL) are cardinal lipases in adipocytes. It is well known that after lipolytic stimulation, HSL is 

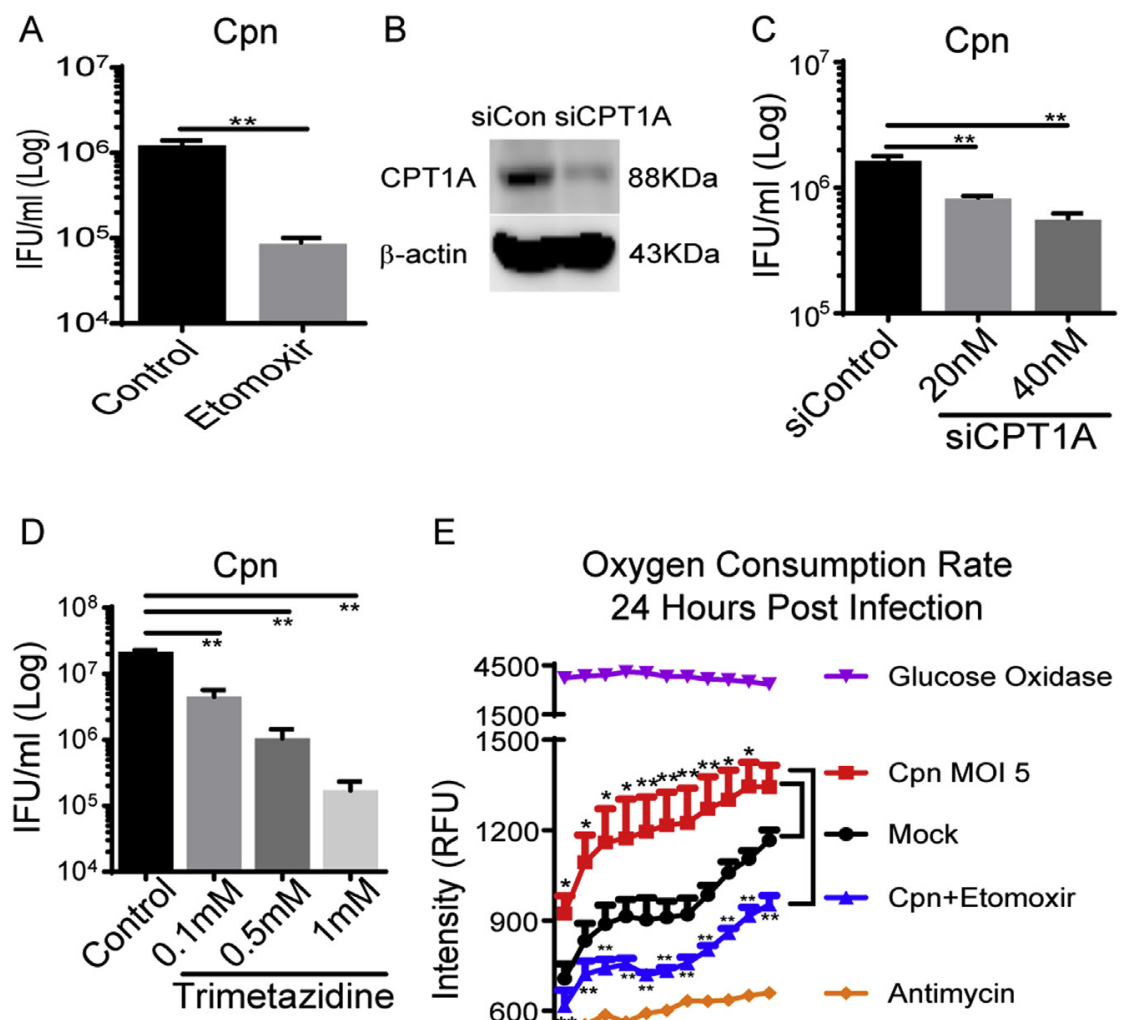

E
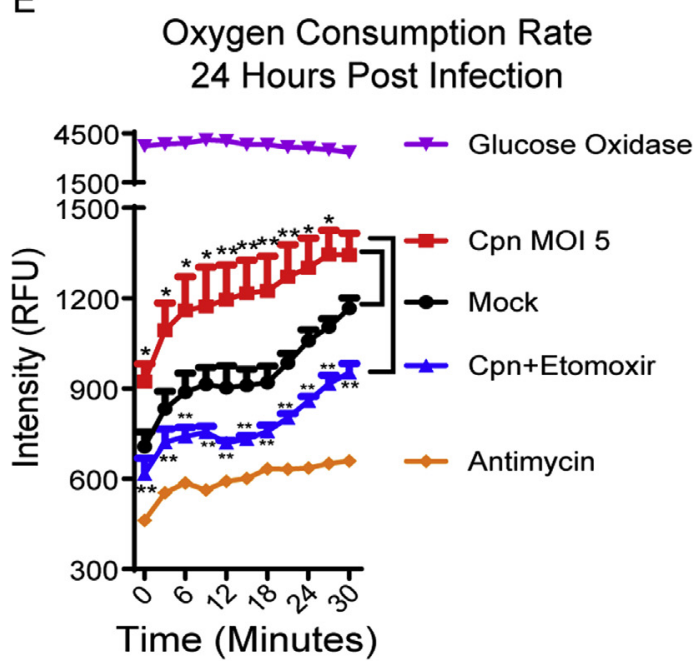

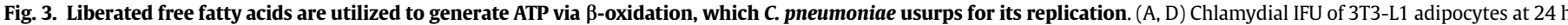

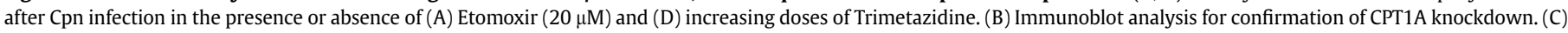

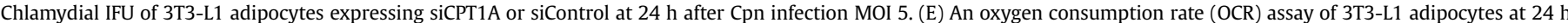

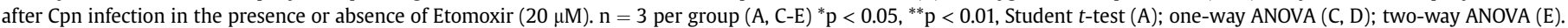

phosphorylated and translocated to lipid droplet surfaces via the cAMP-PKA-HSL signaling pathway [19]. We observed HSL activation in C. pneumoniae-infected adipocytes by Western blot analyses (Fig. 2A). An IFU assay revealed that chemical inhibition of HSL by CAY10499 severely reduced the growth of C. pneumoniae in adipocytes (Fig. 2B). The importance of HSL-mediated lipolysis in intracellular growth of $C$. pneumoniae in adipocytes was confirmed by data acquired using 3T3-L1 cells expressing shRNA against an mRNA Lipe encoding HSL (Fig. 2C and D). Thus, these results clearly demonstrate a crucial role of HSL in C. pneumoniae infectioninduced lipolysis and bacterial growth in murine adipocytes.

In accordance with this, we also found that chemical inhibition of HSL (CAY10499), greatly abrogated infection-induced glycerol release (Fig. 2E). Notably, there was an obvious dissociation between glycerol release and free fatty acids (FFAs) release in C. pneumoniae infection-induced lipolysis (Fig. 2E and F), suggesting that liberated FFAs are utilized intracellularly with reduced secretion, whereas liberated glycerol is secreted without recycling.

\subsection{Liberated FFAs are used to generate ATP via $\beta$-oxidation, which C. pneumoniae usurps for its replication}

Next, we determined how $C$. pneumoniae utilizes liberated FFAs for its growth and survival. Chlamydia is an ATP auxotroph, and decreasing the host's ATP pool can alter pathogen growth. Chlamydiae and Rickettsiae have evolved a remarkable adaptation that enables them to utilize ATP generated by their eukaryotic hosts using specific carrier proteins known as ATP translocases [20-22]. We speculated that liberated FFAs are utilized to generate ATP via fatty acid oxidation (FAO), which serves as an important energy source for $C$. pneumoniae. Therefore, we examined the effect of Etomoxir, a chemical inhibitor of carnitine palmitoyltransferase 1 (CPT-1) that is required for transport of FFAs into mitochondria, the site of $\beta$-oxidation [23]. We found that Etomoxir significantly decreased the intracellular growth of $C$. pneumoniae in adipocytes (Fig. 3A). The role of CPT- 1 on bacterial growth was confirmed by gene silencing of CPT-1A (Fig. 3B) and siCPT-1A treated adipocytes showed decreased intracellular growth of $C$. pneumoniae (Fig. 3C). Furthermore, treatment with trimetazidine [24], a competitive inhibitor of 3-ketoacyl coenzyme A thiolase, a key enzyme in $\beta$ oxidation, greatly diminished the intracellular growth of C. pneumoniae (Fig. 3D). In accordance with these findings, the oxygen consumption rate (OCR) was upregulated in C. pneumoniaeinfected adipocytes (24 hpi) when compared with mock-infected adipocytes (Fig. 3E). This increase in OCR was attenuated by Etomoxir (Fig. 3E). Taken together, these results strongly indicate that liberated FFAs are utilized to generate ATP via FAO, which C. pneumoniae usurps for its replication.

In sharp contrast to C. pneumoniae, CPT-1 did not play a role in the bacterial growth of $C$. muridarum in adipocytes. Chemical inhibition (Etomoxir), gene silencing of CPT-1A, or trimetazidine (FAO inhibitor) did not affect the growth of $C$. muridarum 


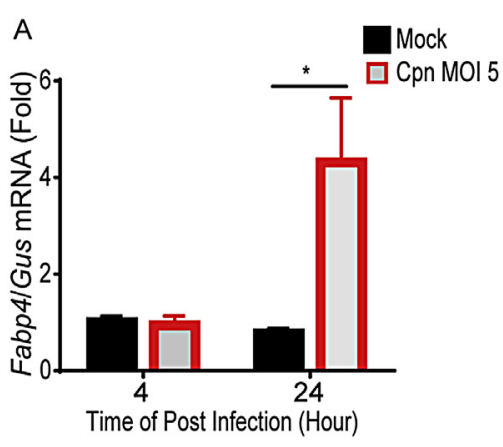

E

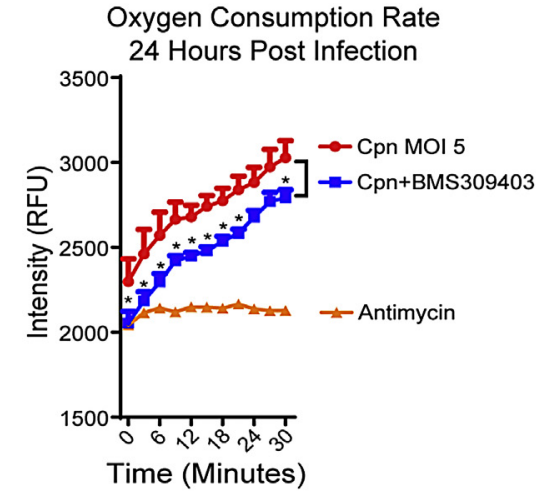

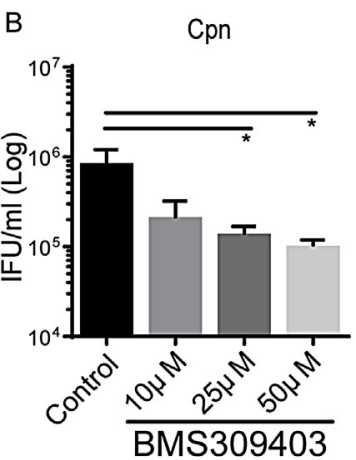

$\mathrm{F}$

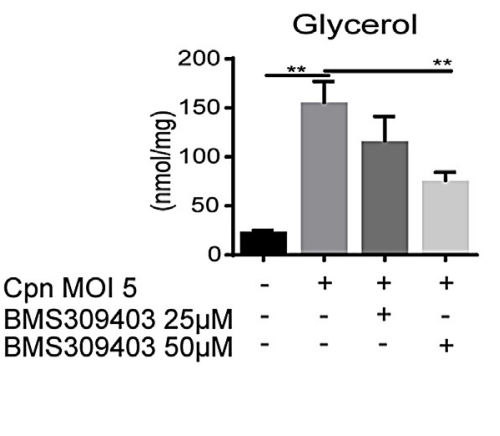

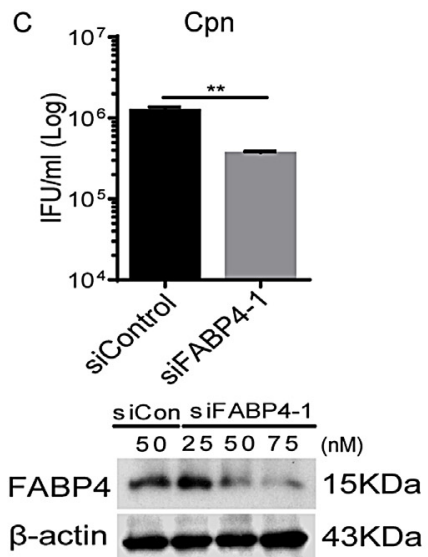

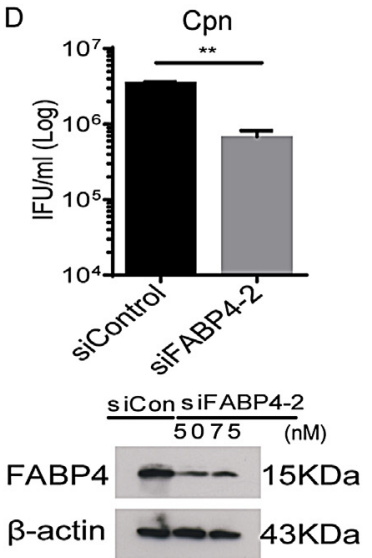

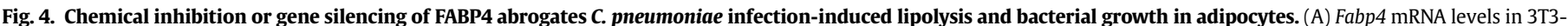

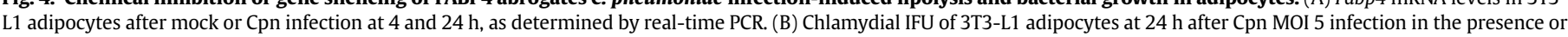

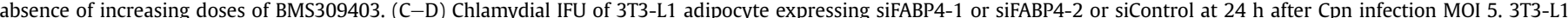

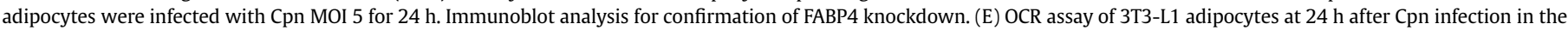

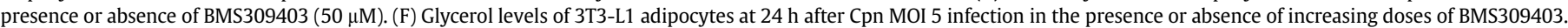

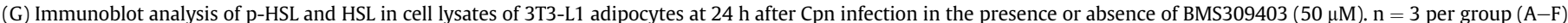
${ }^{*} \mathrm{p}<0.05,{ }^{* *} \mathrm{p}<0.01$, two-way ANOVA (A); one-way ANOVA (B, F); Student $t$-test (C, D); multiple $t$ tests (E).

(Supplementary Fig. 1A C). In fact, the OCR was not elevated in C. muridarum-infected adipocytes when compared with mockinfected cells (Supplementary Fig. 1D). Taken together, these results suggest that $C$. pneumoniae uniquely hijacks host lipid metabolism to obtain ATP provided by FAO of FFAs.

\subsection{Chemical inhibition or genetic silencing of FABP4 abrogates C. pneumoniae infection-induced lipolysis and bacterial growth in adipocytes}

By binding with long-chain fatty acids, FABP4 can affect transportation and $\beta$-oxidation of fatty acids. Therefore we examined the possible role of FABP4 in C. pneumoniae infection-induced lipolysis and subsequent FAO, which $\mathrm{C}$. pneumoniae usurps for its replication. We found that FABP4 mRNA expression was significantly increased upon infection with $C$. pneumoniae when compared with the mock infection in adipocytes (Fig. 4 A). Notably, chemical inhibition of FABP4 by BMS 309403 [5] (Fig. 4 B) or gene silencing (small interfering RNA) of FABP4 significantly decreased the intracellular replication of $C$. pneumoniae in adipocytes (Fig. $4 \mathrm{C}$ and $\mathrm{D}$ ). The $C$. pneumoniae infection-induced increase in OCR was attenuated by FABP4 inhibitor (BMS309403) (Fig. 4 E). Furthermore, chemical inhibition of FABP4 (BMS309403) attenuated C. pneumoniae infection-induced lipolysis (Fig. 4F). This effect was associated with a decrease in phosphorylated HSL, activated form of HSL (Fig. 4G). These findings indicate that FABP4 is functionally linked with HSL-mediated lipolysis in C. pneumoniae-infected adipocytes, which is in line with previous reports showing that basal and stimulated levels of lipolysis are significantly decreased in adipocytes from FABP4 ${ }^{-1-}$ mice [25]. In addition, FABP4 was shown to interact with HSL and facilitate lipolysis and fatty acid transfer $[26,27]$. These results indicate that FABP4 may play important roles both in lipolysis and intracellular transportation of liberated FFAs in C. pneumoniae-infected adipocytes. Furthermore, it was found that chemical inhibition or genetic silencing of FABP4 did not affect the intracellular growth of $C$. muridarum (Supplementary Figs. $1 \mathrm{E}$ and F). Taken together our results indicate that $C$. pneumoniae uniquely exploits FABP4 to reprogram host lipid metabolism for its own benefit, resulting in bacterial growth in adipocytes.

\section{Discussion}

In the present study, we demonstrated that $C$. pneumoniae successfully infects mature adipocytes and exploits host lipid droplet for its replication. Chemical inhibition and genetic manipulation of HSL or FABP4 reduced the intracellular replication of C. pneumoniae in adipocytes. Mechanistically, liberated FFAs were utilized to generate ATP via $\beta$-oxidation, which $C$. pneumoniae usurped for its replication. Chemical inhibitor and gene silencing of FABP4 revealed the importance of FABP4 in HSL-mediated lipolysis and transportation of FFAs to mitochondria. Thus, C. pneumoniae exploits host FABP4 to facilitate fat mobilization and intracellular 
replication in adipocytes. We believe this is a novel finding which highlights the previously unrecognized mechanism of the acquisition of ATP by C. pneumoniae from host cells.

Several pathogens, such as Mycobacterium tuberculosis [13], Rickettsia prowazekii [14] and Trypanosoma cruzi [15], have been shown to target adipose tissues and reside in adipocytes. Our study supports the emerging concept that adipose tissue and adipocytes may be providing a niche or reservoir for intracellular pathogens [16]. We speculate that adipocytes offer a constant intracellular source of nutrients, particularly fatty acids, which can be mobilized from lipid droplets through recruitment of lipases, such as HSL, ATGL, and MGL. In this study, we confirmed the previous pioneering study of infectivity of C. pneumoniae in adipocytes [17] and extended their findings and clarified the mechanistic pathway of how $C$. pneumoniae reprograms host lipid metabolism and facilitates fat mobilization for its own benefit.

Notably, we found that FABP4 plays critical roles both in HSL activation and intracellular mobilization of liberated FFAs to mitochondria for FAO. We found that treatment with a FABP4 inhibitor decreased $C$. pneumoniae infection-induced induction of $\mathrm{p}$-HSL (Fig. 4G), FAO (Fig. 4E), thus resulting in inhibition of $C$. pneumoniae growth in adipocytes (Fig. 4B-D). As an obligate intracellular pathogen, C. pneumoniae needs to obtain nutrients, such as nucleotides (ATP), amino acids, and lipids, from host cells for its replication. Our present findings demonstrate a previously unrecognized mechanism of the acquisition of ATP by C. pneumoniae from its host, i.e., hijacking FABP4 and promoting the liberation of FFAs to generate ATP via the $\beta$-oxidation pathway.

Recently, it was discovered that FABP4 is actively secreted from adipocytes in response to fasting and signals that induce lipolysis such as $\beta$-adrenergic receptor stimulation $[28,29]$ and acts on the liver to stimulate glucose production [30]. In this study we demonstrated that $C$. pneumoniae infection induces the drastic lipolysis in adipocytes. Considering the important pathogenic role of secreted FABP4 in obesity-induced type 2 diabetes and atherosclerosis [5], C. pneumoniae infection-induced FABP4 secretion, if occurs, may have an important in vivo relevance. Therefore, our current results warrant the examination of the hypothesis that FABP4 released from $C$. pneumoniae-infected adipocytes may play a role in C. pneumoniae-induced metabolic pathologies. Further study is required to address this possibility and its significance.

In summary, the present study demonstrates that $C$. pneumoniae infects adipocytes and proliferates by inducing lipolysis and unveils a new mechanism of host lipid metabolism modulation and metabolic pathology caused by $C$. pneumoniae infection. The present study provides a better understanding of the hostC. pneumoniae interaction and identifies the metabolic dependence of intracellular $C$. pneumoniae as a therapeutic target to restrict the growth and persistence of $C$. pneumoniae-related metabolic disorders.

\section{Conflicts of interest}

The authors declare no conflicts of interest associated with this manuscript entitled "Chlamydia pneumoniae exploits adipocyte lipid chaperone FABP4 to facilitate fat mobilization and intracellular growth in murine adipocytes".

\section{Acknowledgements}

NFW is supported by an Indonesia Endowment Fund for Education (LPDP) scholarship from Ministry of Finance, The Republic of Indonesia. This study was supported partly by the grant for Joint Research Project of the Institute of Medical Science, the University of Tokyo to KH (grant number:2016-3014). We would like to thank
Tetsuya Hayashi (Department of Bacteriology, Kyushu University) for critical discussion.

\section{Appendix A. Supplementary data}

Supplementary data related to this article can be found at https://doi.org/10.1016/j.bbrc.2017.11.005.

\section{References}

[1] M. Furuhashi, G.S. Hotamisligil, Fatty acid-binding proteins: role in metabolic diseases and potential as drug targets, Nat. Rev. Drug Discov. 7 (2008) 489-503.

[2] G.S. Hotamisligil, D.A. Bernlohr, Metabolic functions of FABPs-mechanisms and therapeutic implications, Nat. Rev. Endocrinol. 11 (2015) 592-605.

[3] K. Maeda, H. Cao, K. Kono, C.Z. Gorgun, M. Furuhashi, K.T. Uysal, O. Cao, G. Atsumi, H. Malone, B. Krishnan, Y. Minokoshi, B.B. Kahn, R.A. Parker, G.S. Hotamisligil, Adipocyte/macrophage fatty acid binding proteins control integrated metabolic responses in obesity and diabetes, Cell metab. 1 (2005) $107-119$.

[4] E. Erbay, V.R. Babaev, J.R. Mayers, L. Makowski, K.N. Charles, M.E. Snitow S. Fazio, M.M. Wiest, S.M. Watkins, M.F. Linton, G.S. Hotamisligil, Reducing endoplasmic reticulum stress through a macrophage lipid chaperone alleviates atherosclerosis, Nat. Med. 15 (2009) 1383-1391.

[5] M. Furuhashi, G. Tuncman, C.Z. Gorgun, L. Makowski, G. Atsumi, E. Vaillancourt, K. Kono, V.R. Babaev, S. Fazio, M.F. Linton, R. Sulsky, J.A. Robl, R.A. Parker, G.S. Hotamisligil, Treatment of diabetes and atherosclerosis by inhibiting fatty-acid-binding protein aP2, Nature 447 (2007) 959-965.

[6] H. Xu, A.V. Hertzel, K.A. Steen, Q. Wang, J. Suttles, D.A. Bernlohr, Uncoupling lipid metabolism from inflammation through fatty acid binding proteindependent expression of UCP2, Mol. Cell. Biol. 35 (2015) 1055-1065.

[7] M. Furuhashi, R. Fucho, C.Z. Gorgun, G. Tuncman, H. Cao, G.S. Hotamisligil Adipocyte/macrophage fatty acid-binding proteins contribute to metabolic deterioration through actions in both macrophages and adipocytes in mice, J. Clin. investigation 118 (2008) 2640-2650.

[8] A. Xu, Y. Wang, J.Y. Xu, D. Stejskal, S. Tam, J. Zhang, N.M. Wat, W.K. Wong, K.S. Lam, Adipocyte fatty acid-binding protein is a plasma biomarker closely associated with obesity and metabolic syndrome, Clin. Chem. 52 (2006) 405-413.

[9] W.S. Chow, A.W. Tso, A. Xu, M.M. Yuen, C.H. Fong, T.H. Lam, S.V. Lo, H.F. Tse Y.C. Woo, C.Y. Yeung, B.M. Cheung, K.S. Lam, Elevated circulating adipocytefatty acid binding protein levels predict incident cardiovascular events in a community-based cohort: a 12-year prospective study, J. Am. Heart Assoc. 2 (2013), e004176.

[10] M.F. Burak, K.E. Inouye, A. White, A. Lee, G. Tuncman, E.S. Calay, M. Sekiya, A. Tirosh, K. Eguchi, G. Birrane, D. Lightwood, L. Howells, G. Odede, H. Hailu, S. West, R. Garlish, H. Neale, C. Doyle, A. Moore, G.S. Hotamisligil, Development of a therapeutic monoclonal antibody that targets secreted fatty acidbinding protein aP2 to treat type 2 diabetes, Sci. Transl. Med. 7 (2015) 319 ra205.

[11] C.A. Elwell, J.N. Engel, Lipid acquisition by intracellular Chlamydiae, Cell. Microbiol. 14 (2012) 1010-1018.

[12] H.A. Saka, R.H. Valdivia, Acquisition of nutrients by Chlamydiae: unique challenges of living in an intracellular compartment, Curr. Opin. Microbiol. 13 (2010) 4-10.

[13] O. Neyrolles, R. Hernandez-Pando, F. Pietri-Rouxel, P. Fornes, L. Tailleux J.A. Barrios Payan, E. Pivert, Y. Bordat, D. Aguilar, M.C. Prevost, C. Petit B. Gicquel, Is adipose tissue a place for Mycobacterium tuberculosis persistence? PloS one 1 (2006) e43.

[14] Y. Bechah, C.D. Paddock, C. Capo, J.L. Mege, D. Raoult, Adipose tissue serves as a reservoir for recrudescent Rickettsia prowazekii infection in a mouse model, PloS one 5 (2010) e8547.

[15] T.P. Combs, Nagajyothi, S. Mukherjee, C.J. de Almeida, L.A. Jelicks, W. Schubert Y. Lin, D.S. Jayabalan, D. Zhao, V.L. Braunstein, S. Landskroner-Eiger, A. Cordero, S.M. Factor, L.M. Weiss, M.P. Lisanti, H.B. Tanowitz, P.E. Scherer, The adipocyte as an important target cell for Trypanosoma cruzi infection, J. Biol. Chem. 280 (2005) 24085-24094.

[16] H.B. Tanowitz, P.E. Scherer, M.M. Mota, L.M. Figueiredo, Adipose tissue: a safe haven for parasites? Trends Parasitol. 33 (2017) 276-284.

[17] Y. Shi, Y. Liu, A. Murdin, A. Raudonikiene-Mancevski, B.B. Ayach, Z. Yu, I.G. Fantus, P.P. Liu, Chlamydophila pneumoniae inhibits differentiation of progenitor adipose cells and impairs insulin signaling, J. Infect. Dis. 197 (2008) 439-448.

[18] R. Itoh, I. Murakami, B. Chou, K. Ishii, T. Soejima, T. Suzuki, K. Hiromatsu, Chlamydia pneumoniae harness host NLRP3 inflammasome-mediated caspase1 activation for optimal intracellular growth in murine macrophages, Biochem. biophysical Res. Commun. 452 (2014) 689-694.

[19] G.Y. Carmen, S.M. Victor, Signalling mechanisms regulating lipolysis, Cell. Signal. 18 (2006) 401-408.

[20] S. Schmitz-Esser, N. Linka, A. Collingro, C.L. Beier, H.E. Neuhaus, M. Wagner, M. Horn, ATP/ADP translocases: a common feature of obligate intracellular amoebal symbionts related to Chlamydiae and Rickettsiae, J. Bacteriol. 186 
(2004) 683-691.

[21] A. Zomorodipour, S.G. Andersson, Obligate intracellular parasites: Rickettsia prowazekii and Chlamydia trachomatis, FEBS Lett. 452 (1999) 11-15.

[22] O. Trentmann, M. Horn, A.C. van Scheltinga, H.E. Neuhaus, I. Haferkamp Enlightening energy parasitism by analysis of an ATP/ADP transporter from chlamydiae, PLoS Biol. 5 (2007) e231.

[23] J.D. McGarry, N.F. Brown, The mitochondrial carnitine palmitoyltransferase system. From concept to molecular analysis, Eur. J. Biochem./FEBS 244 (1997) $1-14$

[24] P.F. Kantor, A. Lucien, R. Kozak, G.D. Lopaschuk, The antianginal drug trimetazidine shifts cardiac energy metabolism from fatty acid oxidation to glucose oxidation by inhibiting mitochondrial long-chain 3-ketoacyl coenzyme A thiolase, Circulation Res. 86 (2000) 580-588.

[25] N.R. Coe, M.A. Simpson, D.A. Bernlohr, Targeted disruption of the adipocyte lipid-binding protein (aP2 protein) gene impairs fat cell lipolysis and increases cellular fatty acid levels, J. lipid Res. 40 (1999) 967-972.

[26] A.E. Jenkins-Kruchten, A. Bennaars-Eiden, J.R. Ross, W.J. Shen, F.B. Kraemer,
D.A. Bernlohr, Fatty acid-binding protein-hormone-sensitive lipase interaction. Fatty acid dependence on binding, J. Biol. Chem. 278 (2003) 47636-47643.

[27] A.J. Smith, M.A. Sanders, B.R. Thompson, C. Londos, F.B. Kraemer, D.A. Bernlohr, Physical association between the adipocyte fatty acid-binding protein and hormone-sensitive lipase: a fluorescence resonance energy transfer analysis, J. Biol. Chem. 279 (2004) 52399-52405.

[28] S. Kralisch, T. Ebert, U. Lossner, B. Jessnitzer, M. Stumvoll, M. Fasshauer, Adipocyte fatty acid-binding protein is released from adipocytes by a nonconventional mechanism, Int. J. Obes. 38 (2014) 1251-1254.

[29] I. Schlottmann, M. Ehrhart-Bornstein, M. Wabitsch, S.R. Bornstein, V. Lamounier-Zepter, Calcium-dependent release of adipocyte fatty acid binding protein from human adipocytes, Int. J. Obes. 38 (2014) 1221-1227.

[30] H. Cao, M. Sekiya, M.E. Ertunc, M.F. Burak, J.R. Mayers, A. White, K. Inouye, L.M. Rickey, B.C. Ercal, M. Furuhashi, G. Tuncman, G.S. Hotamisligil, Adipocyte lipid chaperone AP2 is a secreted adipokine regulating hepatic glucose production, Cell metab. 17 (2013) 768-778. 